\title{
Plasmonic Dispersion Relations and Intensity Enhancement of Metal-Insulator-Metal Nanodisks
}

\author{
Gernot Schaffernak, Markus K. Krug, ${ }^{\circledR}$ Martin Belitsch, Marija Gašparić, Harald Ditlbacher, \\ Ulrich Hohenester, Joachim R. Krenn, and Andreas Hohenau* ${ }^{*}$
}

Institute of Physics, University of Graz, 8010 Graz, Austria

\section{Supporting Information}

ABSTRACT: We show that the plasmon modes of vertically stacked $\mathrm{Ag}-\mathrm{SiO}_{2}-\mathrm{Ag}$ nanodisks can be understood and classified as hybridized surface and edge modes. We describe their universal dispersion relations and demonstrate that coupling-induced spectral shifts are significantly stronger for surface modes than for edge modes. The experimental data correspond well to numerical simulations. In addition, we estimate optical intensity enhancements of the stacked nanodisks in the range of 1000 .

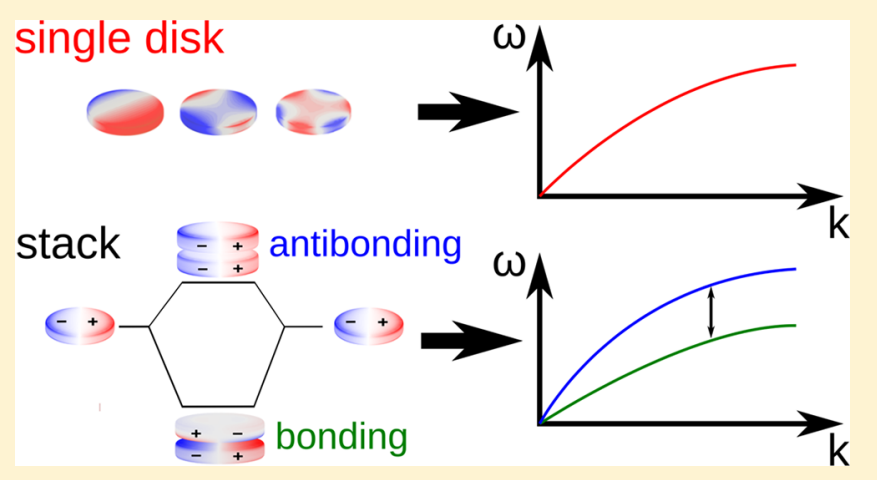

KEYWORDS: metal-insulator-metal, nanoparticle, surface plasmon, surface enhancement

$S_{\text {collec }}$ urface plasmons (SPs) at metal/dielectric interfaces are collective excitations of the free metal electrons, combined with optical near-fields highly confined to the interface. ${ }^{1}$ Particularly strong field confinement is achieved in the vicinity of nanoparticles made from metals as silver, gold, or aluminum, ${ }^{2}$ enabling novel approaches to, for example, sensing. ${ }^{3}$ In this context, a recent roadmap on biosensing emphasizes the importance of coupled plasmonic nanoparticles, that is, particle dimers to enhance optical signals. ${ }^{4}$ In fact, light can be focused into subwavelength volumes in the gap between the particles, inducing strong light-matter interaction, so that even very low analyte concentrations lead to significant spectral modifications of scattered or absorbed light.

A variant of the particle dimer is the vertical stack of two nanoparticles that was studied under different names, as nanosandwiches, ${ }^{5}$ in-tandem pairs, ${ }^{6}$ or metal-insulator-metal $(\mathrm{MIM})^{7}$ structures. MIM nanoparticles are promising structures for sensing applications ${ }^{8}$ for the realization of magnetic field enhancement ${ }^{9}$ and metamaterials with negative index of refraction. ${ }^{10}$ For cylindrical MIM particles, plasmon modes and their hybridization have been well investigated by either electron energy loss spectroscopy ${ }^{5}$ or by cathodoluminescence, ${ }^{11}$ both combined with electron microscopy. In general, the electromagnetic coupling of the two plasmonic constituents of a MIM particle leads to SP hybridization with a splitting of the coupled mode energies. ${ }^{5,12}$ As this splitting depends on the insulator thickness, it is technologically easily controlled. We note in passing that the formal description of modes in nanoparticles is a topic of renewed interest. ${ }^{13}$
In view of a systematic categorization of the plasmon modes of single flat nanoparticles to edge and surface modes ${ }^{14}$ with universal dispersion relations, ${ }^{15}$ the question arises if this concept can be extended to MIM particles. In particular, this concerns the coupling strength of edge and surface modes; are their resulting energy splittings comparable or significantly different? In this Article, we try to answer this by investigating disk-shaped $\mathrm{Ag}-\mathrm{SiO}_{2}-\mathrm{Ag} \mathrm{MIM}$ nanoparticles with systematically varied diameter and $\mathrm{SiO}_{2}$ thickness. In view of the relevance to potential applications in optical sensing, we rely on optical extinction spectroscopy to determine the mode resonance position and strength. Thereby, we avoid the hurdles inherent to STM-EELS or CL investigations when generalizing results to optical measurement schemes.

\section{EXPERIMENTAL SECTION}

Silver nanodisks and MIM disks, that is, stacks of two silver nanodisks separated by a thin $\mathrm{SiO}_{2}$ spacer, are fabricated by electron beam lithography on glass substrates. ${ }^{16}$ A $120 \mathrm{~nm}$ thick poly(methyl-metacrylate) (PMMA) layer, spin-cast on a glass substrate, is used as an electron beam resist and a $\sim 10 \mathrm{~nm}$ aluminum layer is evaporated on top to prevent charging of the surface during electron beam exposure. After aluminum removal with $\mathrm{NaOH}$ and chemical development of the resist, $25 \mathrm{~nm}$ of silver, 5, 10, or $20 \mathrm{~nm}$ of $\mathrm{SiO}_{2}$, and another $25 \mathrm{~nm}$ of silver are thermally evaporated and subsequently removed from the nonexposed areas by a lift-off process in acetone. Immediately after the liftoff, the structures are embedded in a

Received: July 11, 2018

Published: November 13, 2018 
$120 \mathrm{~nm}$ thick PMMA layer to achieve a nearly homogeneous refractive index of the MIM disk environment and to prevent oxidation of the silver.

During the evaporation process, silver accumulates around the edges of the exposed holes in the developed PMMA mask, thereby reducing the size of its openings. As a result, the upper particles in the stack are smaller in diameter than the lower ones. From the analysis of samples fabricated with different insulator heights, we find that the diameter of the upper disk is reduced by $\Delta d=0.77\left(h_{\mathrm{SiO}_{2}}+h_{\mathrm{Ag}}\right)$, with $h$ being the thickness of the respective layers. The MIM disks investigated here have a diameter between 55 and $405 \mathrm{~nm}$ (with an uncertainty of about $5 \mathrm{~nm}$ ), which refers to the diameter of the lower disk (base diameter). Due to the polycrystalline growth of the Ag in combination with the high Ag surface energy, ${ }^{17}$ the MIM disks have nanometric surface roughness and rounded edges. ${ }^{18}$

The MIM disks are arranged in nonperiodic arrays (over an area of $50 \times 100 \mu \mathrm{m}^{2}$ ) to prevent grating effects from modifying the single-particle response. ${ }^{2}$ By maintaining a minimal distance between two nearby particles of 1.25 particle diameters, ${ }^{19}$ we prevent significant near-field coupling between neighboring particles. The SEM images in Figure 1 show an exemplary sample geometry. We note that before SEM imaging the PMMA was removed and the sample was covered with 1 $\mathrm{nm}$ of chromium to avoid electric charging.
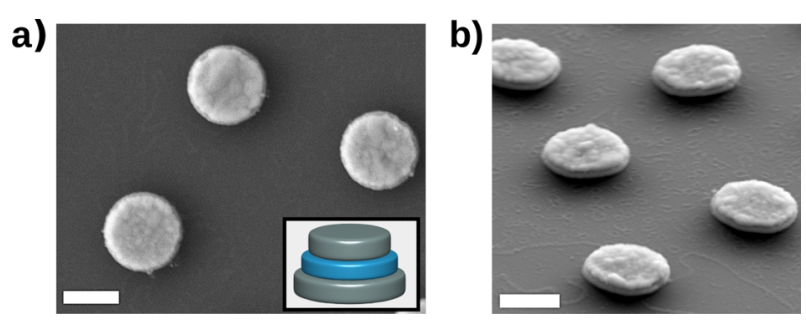

Figure 1. SEM images of a nonperiodic MIM disk array on glass substrate in (a) top view and (b) tilted view. The disks have $405 \mathrm{~nm}$ base diameter and are built from $25 \mathrm{~nm} \mathrm{Ag}, 10 \mathrm{~nm} \mathrm{SiO}$, and $25 \mathrm{~nm}$ Ag. The inset in (a) shows a sketch of the MIM disk geometry. The scale bars are $300 \mathrm{~nm}$.

Optical extinction spectra of the MIM particle arrays are acquired with a microspectrometer (Zeiss MMS1 attached to an optical microscope with a $10 \times$ objective, numerical aperture 0.3 ) over an area about $25 \mu \mathrm{m}$ in diameter $\left(10^{4}-10^{5}\right.$ particles $)$, using the substrate transmission as reference. The spectra are recorded at, first, normal incidence and, second, with the sample tilted at $75^{\circ}$ to the optical axis (corresponding to an angle of incidence of $40^{\circ}$ within the glass/PMMA environment of the MIM disks), for s- and p-polarization (Figure 2a).

\section{RESULTS AND DISCUSSION}

Exemplary extinction spectra of MIM disks with a $\mathrm{SiO}_{2}$ thickness of $20 \mathrm{~nm}$ are depicted in Figure 2b; more spectra are summarized in the Supporting Information, Figure S1. We identify the plasmon modes by comparing the measured data (solid lines) to extinction spectra simulated with the MNPBEM $^{20}$ toolbox (dotted lines) that relies on the boundary element method. The MIM disk geometry is modeled by a stack of two disks of $25 \mathrm{~nm}$ height with a gap height equivalent to that of the $\mathrm{SiO}_{2}$ layer. The disk-edges are rounded with a radius of $5 \mathrm{~nm}$ and the disks environment is assumed to be homogeneous with a refractive index of $n=1.5$, which is close

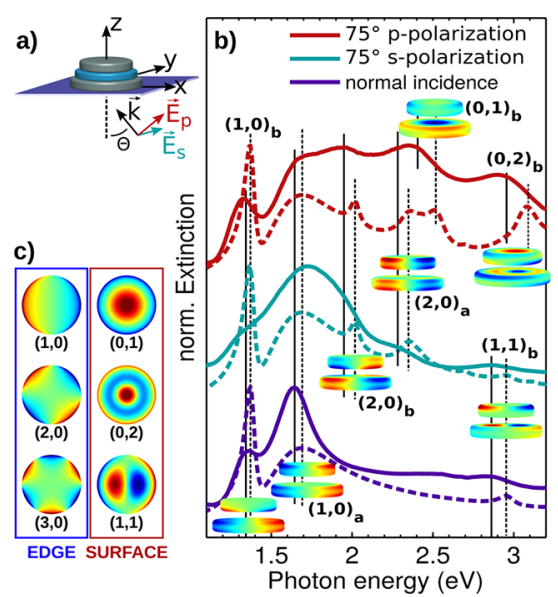

Figure 2. Measurement scheme and plasmon modes of single Ag disks and MIM disks. (a) Sketch of the illumination geometry with an angle of incidence $\Theta$ and the electric field orientations for s- (parallel to $y$ ) and p-polarization (parallel to the $x-z$ plane). The substrate is in the $x$-y-plane. (b) Exemplary experimental (solid lines) and simulated (dashed lines) extinction spectra for a MIM disk with 155 $\mathrm{nm}$ base diameter and a $20 \mathrm{~nm}$ thick $\mathrm{SiO}_{2}$ layer, for normal and inclined $\left(\Theta=75^{\circ}\right)$ incidence. The vertical lines indicate the resonance positions in experiment (solid lines) and simulation (dashed lines). The insets show the simulated charge distributions at the respective resonances. (c) Charge distributions of the plasmon eigenmodes with edge and surface character on a single Ag disk. The mode numbers $(m, n)$ correspond to the numbers $m$ of radial and $n$ of circular node lines. ${ }^{22}$

to the value of $\mathrm{SiO}_{2}(n=1.46$ for substrate and spacer $)$ and PMMA $(n \simeq 1.49)$. The lower disk has the nominal base diameter and the diameter of the upper disk is reduced according to the experimental observations by $\Delta d=0.77\left(h_{\mathrm{SiO}_{2}}\right.$ $\left.+h_{\mathrm{Ag}}\right)$. Due to this size difference of upper and lower disk their resonances do not perfectly overlap, which weakens coupling between modes of the same mode number and partly increases coupling between modes of different mode numbers in upper and lower disk. Both effects contribute to the resulting spectral position of the coupled resonances.

To determine the resonance positions we fit a sum of Lorentzian functions to the experimental spectra and take the simulated resonance positions as the initial guess (interactive peak fitter, ipf.m, ${ }^{21}$ modified to allow for linear background signals).

By simulating the surface charge distributions at the extinction peak maxima, we can assign them to specific modes (Figure $2 b$, insets). In the charge distributions we find on both disks of the MIM system mode pattern analogous to the isolated disk that can be classified as edge and surface modes (compare Figure 2c). Accordingly, we follow the mode nomenclature of ref 22 . The mode numbers $(m, n)$ correspond to the numbers $m$ of radial and $n$ of circular node lines of the calculated charge distributions. We observe dipole $(1,0)$, quadrupole $(2,0)$, and higher order edge modes as well as the breathing (surface) modes $(0,1)$ and $(0,2)$. For the MIM disks, these modes of the single disk split into bonding and antibonding modes. For the bonding mode (subscript $b$ ), the charge distributions on the upper and lower disk have opposite phase and the resonance energy is lowered compared to the single disk. For the antibonding modes (subscript $a$ ), the charge distributions are in phase and the resonance energy is higher; compare the spectra and insets in Figure $2 \mathrm{~b}$. 
All modes show almost unaltered spectral positions, but different peak heights, for normal and inclined incidence in both s- and p-polarization. This is because the excitation efficiencies depend on the polarization and on the phase retardation of the exciting field over the MIM disk, which changes with the angle of incidence. The $(0,1)$ and $(0,2)$ modes can only be excited in p-polarization due to their surface plasmon character. ${ }^{16}$ We note that, in the experimental data, the individual extinction peaks are somewhat broader and slightly shifted compared to the simulations. This is most likely due to averaging over many individual particles with random, nanometric shape variations and the difference in experimental and modeled particle shape and silver permittivity. ${ }^{23}$

We now turn to the dependence of the resonance energies on the particle diameter and again compare the resonances of the MIM disks (Figure $3 \mathrm{~b}$, for $\mathrm{a} \mathrm{SiO}_{2}$ thickness of $20 \mathrm{~nm}$; for 5
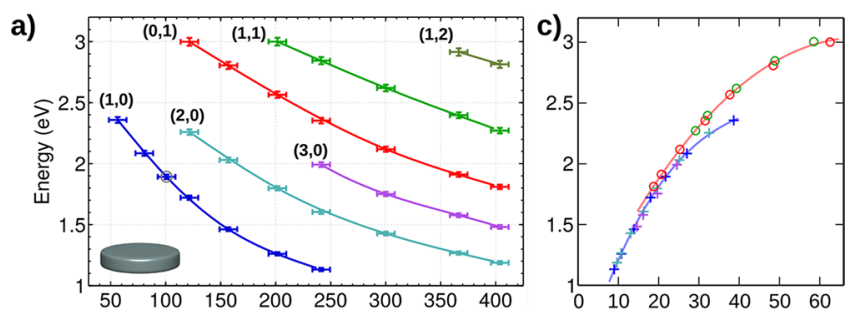

b)
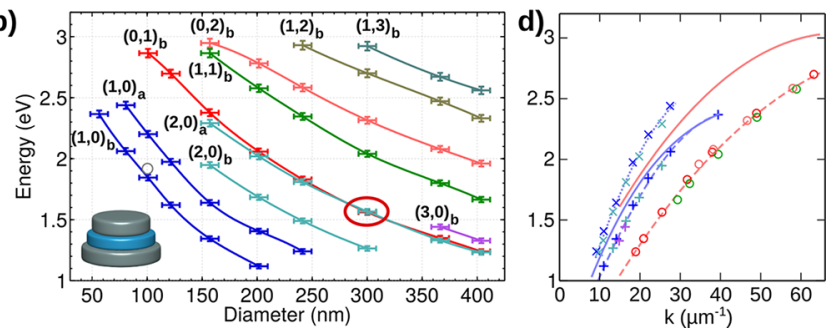

Figure 3. Measured plasmon peak energies and dispersion relations. Base diameter dependent peak energies of (a) single, $25 \mathrm{~nm}$ high Ag disks, (b) MIM disks with an insulator thickness of $20 \mathrm{~nm}$. The modes of the MIM disks are split in bonding and antibonding branches. The red ellipse indicates the mode degeneracy of the $(0,1)_{b}$ and $(2,0)_{a}$ modes. (c, d) Dispersion relations derived from (a) and (b), respectively. Symbols: $\bigcirc$ surface modes, + edge modes and bonding edge modes, and $\times$ antibonding edge modes. The colors of the symbols correspond to those in (a) and (b). All lines are guides to the eye only; the red and blue solid lines in (d) are copied from (b) for reference.

and $10 \mathrm{~nm}$ see the Supporting Information, Figure S2) to the well-known case of single Ag disks (Figure 3a). In general, a red-shift of all resonances is observed for increasing particle diameter. $^{22}$ For the MIM disks, the modes are, in turn, spectrally shifted compared to the single disk and appear in both bonding (subscript $b$ ) and antibonding mode branches (subscript $a$, only observed for the $(1,0),(2,0)$, and $(3,0)$ modes, see below)., ${ }^{5,7}$ From the measured spectral peak energies in Figure $3 \mathrm{a}, \mathrm{b}$, we derive the corresponding dispersion relations, depicted in Figure $3 \mathrm{c}, \mathrm{d}$. We do so by assigning a wavenumber $k$ to the modes, which is determined by the mode number and by the particle circumference (for the edge modes) or diameter (for the surface modes); ${ }^{22}$ for details, see the Supporting Information. As known for the individual nanodisks, this aligns all peak data along two universal dispersion branches, one for edge modes (+ symbols in Figure $3 c)$ and one for surface modes ( $\bigcirc$ symbols).
The corresponding dispersion relations for the MIM disks are plotted in Figure 3d, illustrating the following points. First, just as for individual disks, the MIM disk resonances lie well on diameter-independent dispersion branches that can be attributed to edge or surface modes (Figure 3d). However, second, we find three dispersion branches: bonding edge modes (+ symbols), antibonding edge modes ( $\times$ symbols), and bonding surface modes ( $\bigcirc$ symbols). Antibonding surface modes are not excited efficiently by light for the chosen geometry parameters and are therefore not observed. Third, the surface mode resonances of MIM disks are shifted relative to the single disk case, much stronger than the edge modes resonances. It is likely that this is due to the much higher mode-interaction volume, as the surface modes span the whole disk area compared to the confined edge modes.

The larger shifts are also obvious from the dependence of the spectral resonance position on the insulator thickness (Figure 4). For example, at $300 \mathrm{~nm}$ diameter and $10 \mathrm{~nm}$

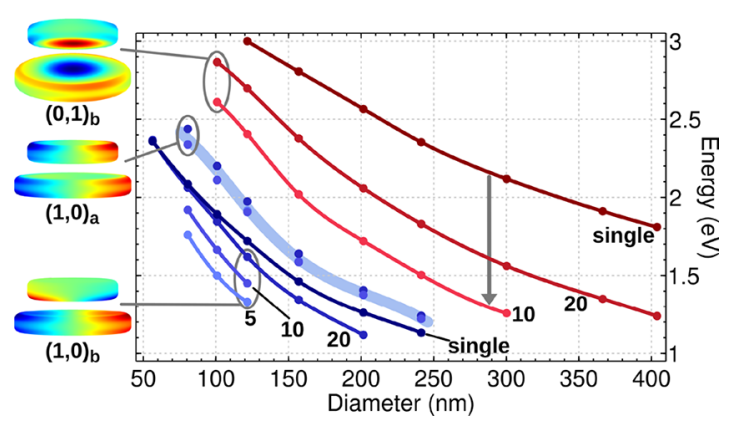

Figure 4. Peak energies of MIM disk plasmon modes in dependence of the base diameter for insulator thicknesses of 5,10 , and $20 \mathrm{~nm}$ (as indicated in the figure), and for the $(1,0)_{a},(1,0)_{b}$ (blue lines), and $(0,1)_{b}$ (red lines) modes (as indicated by the charge pattern; to better illustrate the $(0,1)_{b}$ mode the disks are tilted). The $(1,0)_{a}$ modes follow the same curve (fat, light blue line) for all insulator thicknesses within experimental uncertainty. The lines marked "single" indicate the curves of the $(1,0)$ (blue) and $(0,1)$ (red) modes of the single disk. The gray arrow highlights the large spectral shift of the $(0,1)_{b}$ mode.

insulator thickness, the $(0,1)_{b}$ is red-shifted by $\simeq 0.9 \mathrm{eV}$ (gray arrow), while the shifts of the $(1,0)$ modes are below $0.4 \mathrm{eV}$. This large individual spectral tunability opens new aspects for, for example, using mode degeneracy (for an example, see the modes marked by the red ellipse in Figure $3 b$ ) for achieving directionality of scattering or near-field localization by the coherent excitation of two modes.

Interestingly, the $(1,0)_{a}$ mode follows basically the same diameter dependence, independent of the insulator thickness (thick, light-blue line in Figure 4). In addition, the $(1,0)_{b}$ resonance is nearly not shifted compared to the single Ag disk case for small disk diameters and $20 \mathrm{~nm} \mathrm{SiO}$ thickness. We attribute both effects mainly to the conical shape of the MIM disks. The smaller diameter of the upper $\mathrm{Ag}$ disk causes an overall blue shift of the MIM modes compared to the modes on the single Ag disk of the same base diameter. The cone angle is constant, thus, with decreasing insulator thickness, the upper disk gets larger and the blue shift is reduced. This compensates for the blue shift of the $(1,0)_{a}$ mode caused by increased coupling between upper and lower Ag disk with decreasing insulator thickness. 
With respect to potential application of MIM structures in surface-enhanced spectroscopy, we find that by changing the insulator thickness and particle diameter, the resonance position of a particular mode can be conveniently tuned within a particularly large spectral range. We now complement this by asking for the achievable optical near-field enhancements of the individual modes, aiming at high enhancement values. To analyze and compare the near-field enhancement of edge and surface modes, we concentrate on the lowest order modes (i.e., the bonding and antibonding dipolar $(1,0)$ and the bonding breathing $(0,1)$ modes $)$, as they couple most efficiently to light. In the following we discuss results from simulations with the MNPBEM toolbox for homogeneous environment with a refractive index of 1.5 and a thickness of the dielectric gap of $15 \mathrm{~nm}$ (Figure 5). a)

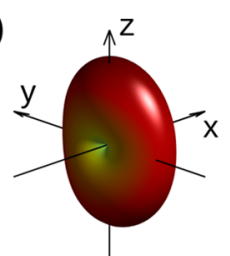

$(1,0)$

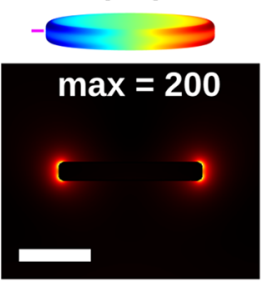

d)

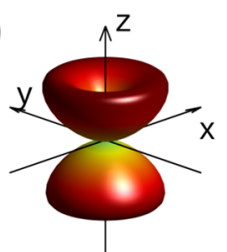

$(0,1)$

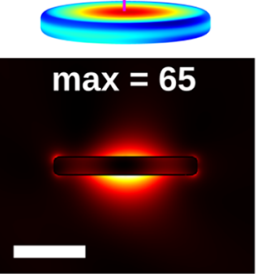

b)

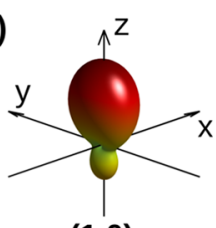

$(1,0)_{b}$
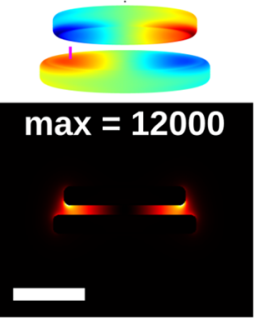

e)
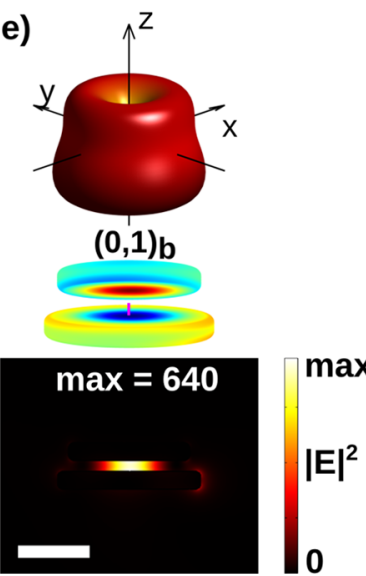

c)

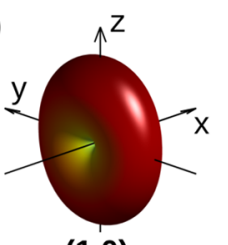

$(1,0) a$
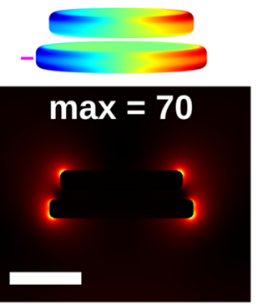

Figure 5. Emission diagrams for excitation by a local oscillating point dipole (top), charge distributions (middle; the short purple line indicates dipole position and orientation), and optical near-field intensities in the $x-z$-plane (bottom) for plane-wave excitation: (a) the $(1,0)$ mode of single disk; $(b)$ the $(1,0)_{b}$ mode, and (c) the (1, $0)_{a}$ mode of a MIM disk; (d) the $(0,1)$ mode of a single disk; (e) the $(0,1)_{b}$ mode of a MIM disk. Ag thickness is $25 \mathrm{~nm}$ and the insulator thickness is $15 \mathrm{~nm}$. For the simulations, a homogeneous environment of refractive index 1.5 was assumed.

Before addressing the actual values of optical near-field enhancement, we have to choose a suitable excitation geometry. Due to the specific charge pattern of the different modes, directional and polarization dependencies are expected. To find the optimum excitation geometry for each mode, we simulate first the emission diagrams of the MIM disks, assuming their excitation by an oscillating point dipole. The dipoles are positioned and polarized to match the near fields in regions of large enhancement. As deduced from the simulated charge distributions in Figure 5, this is for the $(1,0)$ mode of the single disk, (a), $x$-polarized at the disk rim; for the $(1,0)_{b}$ mode, (b), $z$-polarized in the gap below the rim of the upper disk; for the $(1,0)_{a}$ mode, $(c), x$-polarized at the rim of the lower disk; for the $(0,1)$ mode of the single disk, (d), $z$ polarized at the disk center; for the $(0,1)_{b}$ mode, (e), $z$ polarized in the gap at the disk center (details: Supporting Information, Figure S3). The emission diagrams are to be understood as an approximation to those of the corresponding modes. This is justified if (a) the direct emission from the point dipoles used as source is small compared to that of the MIM disk and (b) the emission from the MIM disk is dominated by one excited mode. For the above cases, both are reasonably well fulfilled. According to optical reciprocity, these diagrams also represent the directional dependence of the excitation efficiency of the dipole with a plane wave.

Comparing single and MIM disks, we find that the emission diagrams for the antibonding dipolar mode (Figure 5c) resembles that of the dipolar single disk mode (Figure 5a), while the bonding dipolar mode (Figure $5 b$ ) strongly deviates. This is because for the $(1,0)_{a}$ mode both disk dipoles are in phase, while they show nearly opposite phase for the $(1,0)_{b}$ mode, interfering destructively in the $y$-direction. The obvious asymmetry in $z$-direction is related to the difference in diameter between upper and lower disks. A particularly strong modification of the emission diagram of the MIM disk compared to a single disk is observed for the $(0,1)$ mode. For the single disk, the emission diagram (Figure 5d) resembles the one expected for an extended radial dipole arrangement in the $x-y$-plane. However, for the $(0,1)_{b}$ mode the charge distributions and net dipole moments from the upper and lower disk have nearly opposite phase and the charge distribution is strongly confined to the gap region, which finally leads to a strongly distorted emission diagram (Figure 5e).

To evaluate the optical near-field enhancement under plane wave excitation, the excitation direction was chosen parallel to a direction of maximum scattering in the $x-z$-plane as calculated above. We start with the dipolar $(1,0)_{a}$ mode, which has the largest dipole moment and thus shows the strongest signature in the experimental extinction spectra (Figure 2). However, it turns out that due to the symmetric charge distribution on both disks, the optical near fields in the gap are actually reduced, compared to the rim of the disks or even the single disk case (see Figure 5a,c). Strong field enhancements in the gap region appear only for the bonding modes with antisymmetric charge distribution on both disks (Figure 5b,e). The maximum intensity enhancement in the midgap region calculated at the respective resonance is about 640 for the $(0,1)_{b}$ mode and 12000 for the $(1,0)_{b}$ mode. The latter value is particularly promising but probably hard to realize, as the simulated extinction peak is narrower and significantly stronger than observed in the experiment. The reason for this discrepancy could be related to a strong sensitivity of this mode to random structural deviations from the idealized shape. Nonetheless, intensity enhancements in the range of 1000 seem achievable even for the relatively large insulator thickness of $15 \mathrm{~nm}$ considered here. We note that the accessibility of the insulator region by an analyte necessary for applications in surface-enhanced spectroscopy and sensing 
could be achieved by the use of nanoporous insulators or the lateral under-etching of the insulator. ${ }^{24}$

\section{CONCLUSION}

By means of optical spectroscopy and numerical simulations, we investigated the plasmonic modes of MIM disks that are dominated by the hybridization of the single disk modes. The MIM disk modes have either surface or edge character and align to universal dispersion relations, which are, however, split into bonding and antibonding branches. The spectral resonance shift upon hybridization is found to be much larger for surfaces modes than for edge modes and it can be tuned by the insulating layer thickness. Consequently, we demonstrate that with a proper choice of diameter and insulator thickness, selected edge and surface modes can be designed to energetic degeneracy. This could be applied to tailor specific optical near-field profiles and emission diagrams, ${ }^{25}$ for example, to improve the directional emission of fluorescent molecules for sensor or quantum optics applications. The strongest optical near-field enhancements of MIM disk modes is found for the bonding modes with antisymmetric charge distributions on both disks. For a gap thickness of $15 \mathrm{~nm}$, the expected intensity enhancement is about 1000, which is promising for applications in surface-enhanced spectroscopies.

\section{ASSOCIATED CONTENT}

\section{S Supporting Information}

The Supporting Information is available free of charge on the ACS Publications website at DOI: 10.1021/acsphotonics.8b00938.

Experimental and simulated extinction spectra, insulator thickness dependence of the resonances, details on how to derive the dispersion relations from the experimental data, and details of the optical near-field enhancement calculations (PDF).

\section{AUTHOR INFORMATION}

\section{Corresponding Author}

*E-mail: andreas.hohenau@uni-graz.at.

\section{ORCID}

Markus K. Krug: 0000-0002-3563-1721

Andreas Hohenau: 0000-0002-9034-3044

\section{Notes}

The authors declare no competing financial interest.

\section{ACKNOWLEDGMENTS}

The authors acknowledge the financial support from the Austrian Science Fund (FWF): P25034-N20.

\section{REFERENCES}

(1) Barnes, W. L.; Dereux, A.; Ebbesen, T. W. Surface plasmon subwavelength optics. Nature 2003, 424, 824-830.

(2) Maier, S. A.; Atwater, H. A. Plasmonic: Localization and guiding of electromagnetic energy in metal/dielectric structures. J. Appl. Phys. 2005, 98, No. 011101.

(3) Anker, J. N.; Hall, W. P.; Lyandres, O.; Shah, N. C.; Zhao, J.; Duyne, R. P. V. Biosensing with plasmonic nanosensors. Nat. Mater. 2008, 7, 442-453.

(4) Fabrizio, E. D.; Schlücker, S.; Wenger, J.; Regmi, R.; Rigneault, H.; Calafiore, G.; West, M.; Cabrini, S.; Fleischer, M.; van Hulst, N. F.; Garcia-Parajo, M. F.; Pucci, A.; Cojoc, D.; Hauser, C. A. E.; Ni, M.
Roadmap on biosensing and photonics with advanced nano-optical methods. J. Opt. 2016, 18, No. 063003.

(5) Dmitriev, A.; Pakizeh, T.; Käll, M.; Sutherland, D. S. Gold-SilicaGold Nanosandwiches: Tunable Bimodal Plasmonic Resonators. Small 2007, 3, 294-299.

(6) Ekinci, Y.; Christ, A.; Agio, M.; Martin, O. J. F.; Solak, H. H.; Löffler, J. F. Electric and magnetic resonances in arrays of coupled gold nanoparticle in-tandem pairs. Opt. Express 2008, 16, 1328713295.

(7) Verre, R.; Yang, Z. J.; Shegai, T.; Kall, M. Optical Magnetism and Plasmonic Fano Resonances in Metal-Insulator-Metal Oligomers. Nano Lett. 2015, 15, 1952-1958.

(8) Horrer, A.; Krieg, K.; Freudenberger, K.; Rau, S.; Leidner, L.; Gauglitz, G.; Kern, D. P.; Fleischer, M. Plasmonic vertical dimer arrays as elements for biosensing. Anal. Bioanal. Chem. 2015, 407, 8225-8231.

(9) Pakizeh, T.; Abrishamian, M. S.; Granpayeh, N.; Dmitriev, A.; Käll, M. Magnetic-field enhancement in gold nanosandwiches. Opt. Express 2006, 14, 8240-8246.

(10) Shalaev, V. M.; Cai, W.; Chettiar, U. K.; Yuan, H.; Sarychev, A. K.; Drachev, V. P.; Kildishev, A. V. Negative index of refraction in optical metamaterials. Opt. Lett. 2005, 30, 3356-3358.

(11) Kuttge, M.; de Abajo, F. J. G.; Polmann, A. Ultrasmall Mode Volume Plasmonic Nanodisk Resonators. Nano Lett. 2010, 10, 15371541.

(12) Yankovich, A. B.; Verre, R.; Olsén, E.; Persson, A. E. O.; Trinh, V.; Dovner, G.; Käll, M.; Olsson, E. Multidimensional Hybridization of Dark Surface Plasmons. ACS Nano 2017, 11, 4265-4274.

(13) Powell, D. A. Interference between the Modes of an AllDielectric Meta-atom. Phys. Rev. Appl. 2017, 7, No. 034006.

(14) Schmidt, F.-P.; Ditlbacher, H.; Hohenester, U.; Hohenau, A.; Hofer, F.; Krenn, J. R. Universal dispersion of surface plasmons in flat nanostructures. Nat. Commun. 2014, 5, na.

(15) Kuttge, M.; Cai, W.; García de Abajo, F. J.; Polman, A. Dispersion of metal-insulator-metal plasmon polaritons probed by cathodoluminescence imaging spectroscopy. Phys. Rev. B: Condens. Matter Mater. Phys. 2009, 80, No. 033409.

(16) Krug, M. K.; Reisecker, M.; Hohenau, A.; Ditlbacher, H.; Trügler, A.; Hohenester, U.; Krenn, J. R. Probing plasmonic breathing modes optically. Appl. Phys. Lett. 2014, 105, 171103.

(17) Tinguely, J.-C.; Sow, I.; Leiner, C.; Grand, J.; Hohenau, A.; Felidj, N.; Aubard, J.; Krenn, J. R. Gold Nanoparticles for Plasmonic Biosensing: The Role of Metal Crystallinity and Nanoscale Roughness. BioNanoScience 2011, 1, 128-135.

(18) Haberfehlner, G.; Schmidt, F.-P.; Schaffernak, G.; Hörl, A.; Trugler, A.; Hohenau, A.; Hofer, F.; Krenn, J. R.; Hohenester, U.; Kothleitner, G. Imaging of Gap Plasmons in Vertically Coupled Nanoparticles by EELS Tomography. Nano Lett. 2017, 17, 67736777.

(19) Rechberger, W.; Hohenau, A.; Leitner, A.; Krenn, J. R.; Lamprecht, B.; Aussenegg, F. R. Optical properties of two interacting gold nanoparticles. Opt. Commun. 2003, 220, 137-141.

(20) Hohenester, U.; Trügler, A. MNPBEM - A Matlab toolbox for the simulation of plasmonic nanoparticles. Comput. Phys. Commun. 2012, 183, 370-381.

(21) O'Haver, T. C. Interactive Peak Fitter (ipf.m). http:// terpconnect.umd.edu/toh/spectrum/SignalProcessingTools.html.

(22) Schmidt, F.-P.; Ditlbacher, H.; Hohenester, U.; Hohenau, A.; Hofer, F.; Krenn, J. R. Dark plasmonic breathing modes in silver nanodisks. Nano Lett. 2012, 12, 5780.

(23) Johnson, P. B.; Christy, R. W. Optical Constants of the Noble Metals. Phys. Rev. B 1972, 6, 4370-4379.

(24) Dai, F. Surface Enhanced Raman Scattering (SERS) Based on Resonant Gap Modes of Vertical Metal-Insulator-Metal (MIM) Structures. Presented at ICES 2017, Munich, Germany, September, 2017.

(25) Coenen, T.; Arango, F. B.; Koenderink, A. F.; Polman, A. Directional emission from a single plasmonic scatterer. Nat. Commun. 2014, 5, 3250. 